\title{
METODOLOGÍA PARA EL DESARROLLO DE PROCESOS DE AUTOMATIZACIÓN SEGÚN LA NORMA ANSI/ISA-88
}

\author{
A. Burgos \\ Departamento de Ingeniería de Sistemas y Automática, Escuela de Ingeniería de Bilbao, UPV/EHU \\ arantzazu.burgos@ehu.eus \\ M.L. Alvarez, I. Sarachaga, M. Marcos \\ Departamento de Ingeniería de Sistemas y Automática, Escuela de Ingeniería de Bilbao, UPV/EHU \\ marialuz.alvarez@ehu.eus, isabel.sarachaga@ehu.eus,marga.marcos@ehu.eus
}

\begin{abstract}
Resumen
La estandarización es clave en la puesta en marcha de Industry 4.0 donde las normas internacionales existentes constituyen el punto de partida para el desarrollo de los nuevos estándares demandados. En el ámbito de la automatización industrial se propone el uso del estándar ANSI/ISA-88 para su aplicación en fabricación discreta, procesos continuos e incluso en logística. En el presente trabajo se propone una extensión de la metodología MeiA (Methodology for industrial Automation systems) concebida para desarrollar software de control en el ámbito de la ingeniería de automatización. Dicha extensión consiste en la incorporación del estándar ANSI/ISA88. La aplicabilidad de la extensión propuesta se ilustra mediante un caso de estudio, que pone de manifiesto la obtención de los modelos propuestos por ANSI/ISA S88 siguiendo la metodología, así como la descripción de los modos de operación de acuerdo a dicho estándar.
\end{abstract}

Palabras Clave: Industry 4.0, automatización, metodología MeiA., ANSI/ISA-88.

\section{INTRODUCCIÓN}

La adaptación de las empresas a la Cuarta Revolución Industrial precisa que se integren las nuevas tecnologías de la información en los procesos productivos, se incrementen los niveles de automatización y la industria se vuelve más inteligente.

La estandarización es clave en la puesta en marcha de la denominada Industry 4.0 para facilitar el desarrollo y la implantación de las tecnologías que sustentan la transformación digital, y garantizar la interoperabilidad $\mathrm{y}$ conectividad de diferentes sistemas y soluciones. Pero no es suficiente una estandarización a nivel regional o nacional; se precisa que la estandarización sea a nivel internacional [13].
En este sentido, [3] presenta el estado actual de los estándares relevantes en el ámbito de Industry 4.0, un esbozo de las necesidades actuales de normalización para acometer dicho reto y recomendaciones detalladas a tener en cuenta en el desarrollo de nuevos estándares, que sirvan a las partes involucradas de los distintos sectores tecnológicos (la automatización, la tecnología de la información y las comunicaciones, y la tecnología de fabricación). En cualquier caso, las normas internacionales existentes constituyen el punto de partida para el desarrollo de los nuevos estándares demandados por Industry 4.0.

En el ámbito de la automatización industrial, el informe apunta que existen numerosas normas que han demostrado su valía en la práctica, pero precisan de actualizaciones, extensiones o reorganizaciones sustanciales para conseguir normas más compactas, más robustas y con menos solapamientos que satisfagan los nuevos requisitos. Así, el informe propone el uso del estándar ANSI/ISA-88 (S88) [8] en lo que respecta al proceso productivo, señalando que, aunque tiene sus orígenes en la ingeniería de procesos por lotes, su metodología es tan estructurada que su aplicación en fabricación discreta, procesos continuos e incluso en logística tiene un gran potencial aún sin explotar. Asimismo, consideran que los conceptos metodológicos fundamentales de Industry 4.0 con modelos de flujo de materiales y fórmulas individuales de ensamblado son similares a las de los procesos por lotes.

Por los anteriores motivos, en el presente trabajo se propone una extensión de la metodología MeiA. (Methodology for industrial Automation systems) [15] concebida para desarrollar software de control en el ámbito de la ingeniería de automatización. Dicha extensión consiste en la incorporación de la descripción de los distintos modos de operación de acuerdo al estándar ANSI/ISA S88, que se suma a la realizada según la guía GEMMA [1], para establecer los requisitos de los sistemas de control industrial. Además, se establece la relación entre los modelos 
obtenidos siguiendo la metodología y los modelos propuestos por ANSI/ISA S88.

La estructura del artículo es la siguiente: en el apartado 2 se realiza una revisión de los estándares más utilizados para la definición de requisitos de los sistemas de control industrial y sus relaciones. El apartado 3 presenta la extensión de la metodología MeiA, con el fin de incorporar el estándar ANSI/ISA S88. El apartado 4 describe el caso de estudio realizado en la célula de ensamblaje DISA FMS-200. Finalmente, las conclusiones se exponen en el apartado 5.

\section{DEFINICIÓN DE REQUISITOS DE LOS SISTEMAS DE CONTROL INDUSTRIAL}

Cualquier proceso de desarrollo de software comienza con la especificación de los requisitos funcionales $\mathrm{y}$ no funcionales que establecen el comportamiento deseado del mismo. Unas especificaciones incompletas o incorrectas pueden implicar errores o, al menos, resultados inesperados [9]. Con objeto de asegurar la correcta captura y definición de requisitos para los sistemas de control industrial, estos requisitos se describen mediante los modos de operación.

La estrategia de control no sólo debe contemplar el simple funcionamiento automático, sino que debe abordar también las situaciones de fallo, de parada de emergencia, los procesos de rearme y puesta en marcha, las marchas de test, el control manual... Por lo tanto, la estrategia de control se complica al abordar distintos modos de operación con los consiguientes cambios de modo, y además, cada modo de operación debe contemplar todos los posibles estados que puede presentar el proceso de producción automatizado y las transiciones entre dichos estados.

Este incremento en la complejidad de la estrategia de control precisa el uso de guías de diseño basadas en modos de operación que permitan afrontar los problemas derivados de la implementación de los distintos modos de operación. En [17] se realiza una comparativa de las tres guías de diseño basadas en modos de operación: GEMMA de ADEPA, S88 de ISA y PackML de OMAC. Destaca la generalidad de la guía GEMMA que permite guiar los procesos de desarrollo de cualquier sistema automatizado, mientras que S-88 está orientado a los sistemas de control por lotes y PackML a la automatización de maquinaria de envasado y embalaje. Pero incide en la dificultad de integrar GEMMA en un proceso de desarrollo automático al tratarse de un proceso manual.
En los siguientes sub-apartados se presenta la guía GEMMA que es la utilizada por la metodología MeiA, S88 que es el estándar propuesto en el ámbito de Industry 4.0, y una tabla que relaciona los estados de S88 los estados de GEMMA.

\subsection{GEMMA (Guide des Modes d'Etude et d'Arrêts Marches)}

Desarrollada por ADEPA (Agence pour le Developpement of Appliquée Productique), GEMMA es una guía genérica para la identificación sistemática de los posibles estados en los que se puede encontrar cualquier proceso automatizado desde una perspectiva de control, distinguiendo entre control con y sin alimentación. Cuando la alimentación está presente, los posibles estados se agrupan en tres familias: la familia $\mathrm{F}$ para los procedimientos de funcionamiento, la familia A para los procedimientos de paro y la familia D para los procedimientos de fallo.

Su notación gráfica presenta las relaciones entre los estados de las distintas familias. Los estados se representan mediante rectángulos, las transiciones entre estados se representan mediante líneas orientadas, y la evolución entre estados está supeditada al cumplimiento de las condiciones que acompañan a las líneas orientadas. Dado que el gráfico sólo muestra las transiciones más usuales, será necesario añadir aquellas transiciones requeridas por una aplicación particular, usando líneas orientadas sin procedencia para representar la evolución a un estado desde todos los demás.

Para su aplicación, GEMMA recomienda un proceso que comienza por determinar los posibles estados del proceso, para identificar posteriormente las transiciones entre estados con las condiciones asociadas. Este análisis proporciona valiosa información sobre el comportamiento del proceso, los límites del sistema de control y las interfaces con el entorno.

Varios autores han trabajado combinando GEMMA y GRAFCET [2]. Por ejemplo, [16] propone la implementación del ciclo de producción con GRAFCET como punto de partida para aplicar GEMMA con el fin de desarrollar sistemas de automatización. [5] combina el diagrama de casos de uso de UML [11], GEMMA y GRAFCET en una metodología orientada a objetos para sistematizar el modelado de sistemas secuenciales de eventos discretos. [12] traduce el GEMMA asociado al comportamiento del sistema a un SFC (Sequential Function Chart) [7] de alto nivel y cada estado GEMMA se traduce a SFCs de bajo nivel, logrando la sincronización a través de la coordinación vertical. 
La generalidad de su ámbito de aplicación y la proximidad al vocabulario del personal de planta fueron los aspectos que permitieron identificar la guía GEMMA como la candidata adecuada para la fase de análisis de requisitos de la metodología MeiA.

\subsection{ANSI/ISA S88 - BATCH CONTROL}

El estándar S88 proporciona la terminología y los modelos de referencia para definir los requisitos de control de las plantas de fabricación por lotes, mejorando la comunicación entre las partes involucradas.

El modelo de proceso es un modelo jerárquico que proporciona un mayor nivel de detalle del proceso dividiendo éste en una o varias etapas, las etapas en una o varias operaciones y estas últimas en una $o$ varias acciones.

El modelo físico organiza jerárquicamente los sistemas de la empresa en sitios (subdivisión lógica, operativa, geográfica o física), áreas, células de proceso, unidades, módulos de equipo y módulos de control. Los tres primeros niveles están fuera del ámbito del control y, por tanto, no se definen sus límites en este estándar. Las características de los restantes niveles se corresponden exactamente con los niveles del modelo de entidad de equipo.

El modelo de entidad de equipo organiza jerárquicamente las entidades equipo (formadas por la combinación del equipo físico y su control) en células de proceso que incluyen unidades, módulos de equipo y módulos de control.

El modelo de control procedimental es un modelo jerárquico que acomete las tareas de un proceso completo o parte de un proceso en base a recursos de una célula de proceso específica. Cada procedimiento organiza el orden de ejecución de uno o varios procedimientos de unidad, éstos especifican el orden de ejecución de una o varias operaciones, y estas últimas de una o varias fases.

El estándar también introduce el concepto de receta, definiendo ésta como la información que define un requisito de fabricación para un producto específico, intermedio o cambio de estado del equipo, es decir, las recetas describen los productos y cómo son producidos. No obstante, la norma identifica cuatro tipos de recetas: General, Sitio, Maestra y Control. Mientras las recetas General y de Sitio describen las técnicas de los procesos de producción independientemente de los equipos, las recetas Maestra y de Control definen los procedimientos que implementan los procesos con los recursos físicos reales dependiendo del equipo en una célula de procesos concreta. Las recetas contienen las siguientes categorías de información: cabecera, fórmula, requisitos de equipo, procedimiento y otra información.

El procedimiento de la receta define la estrategia para llevar a cabo un proceso. Los procedimientos de las recetas General y Sitio están estructurados usando los niveles descritos en el modelo de proceso, lo cual permite describir el proceso sin considerar los equipos. Sin embargo, los procedimientos de las recetas Maestra y Control están estructurados usando los niveles descritos en el modelo de control procedimental $\mathrm{y}$, por tanto, están relacionados con los equipos.

En cuanto a los modos y estados de operación, la norma los define para las entidades equipo y los elementos procedimiento. En los modelos que los describen, las transiciones entre los elementos procedimiento o entre las entidades equipo ocurren dentro de cada nivel jerárquico. Los modos especifican la manera en que las transiciones tienen lugar; los estados especifican su estado actual.

A modo de ejemplo, S88 utiliza tres modos (automático, semiautomático y manual) para los elementos procedimiento, y dos modos (automático y manual) para las entidades equipo. En el caso de los elementos procedimiento, el modo establece la manera de progresar el procedimiento y quién puede intervenir en dicha progresión, determinando cómo se manejan las transiciones en el modelo de estados: en el modo automático, las transiciones se llevan a cabo sin interrupción cuando se cumplen las condiciones de transición; en el modo semiautomático, se requiere aprobación manual para realizar la transición (una vez se cumplen las condiciones de transición), permitiendo omitir o volver a ejecutar uno o más procedimientos sin cambiar su orden; en el modo manual, los elementos procedimiento y su orden de ejecución se especifican manualmente.

En el caso de las entidades equipo, el modo determina cómo pueden ser manipulados sus estados. Las entidades equipo en modo automático son manipuladas por sus algoritmos de control, mientras que en el modo manual son manipuladas por un operador.

El número de posibles estados y sus nombres varían dependiendo de los requisitos de la aplicación. A modo de ejemplo, S88 utiliza un conjunto de estados y comandos de evolución entre estados diferente para los elementos procedimiento y las entidades equipo. Para los primeros, los estados incluyen Running, Holding, Stopped, Paused, Aborted y Complete, siendo los comandos aplicables Start, Hold, Pause, 
Stop y Abort. Para los segundos, los estados incluyen On, Off, Closed, Opened, Travelling, Tripped, y 35\% Open, siendo los comandos aplicables Start, Stop, Reset, Open, Close y Open 35\%.

Tanto un cambio de modo como un cambio de estado tiene lugar sólo cuando se cumplen las condiciones para el cambio, bien por la lógica interna del sistema o por un comando.

El conjunto completo de estados, comandos y transiciones permitidas para cada elemento procedimiento constituye su modelo de estados procedimental, que se representa en el diagrama de transición de estados. Dicho diagrama incluye dos tipos de estados procedimentales: estados cuyo nombre finaliza con "ing" en los que el elemento procedimiento orquesta un conjunto definido de acciones, y estados en los que no se permite realizar ninguna acción inmediata.

S88 presenta un modelo de estados procedimental de referencia completo y una versión reducida del mismo que será suficiente en la mayoría de los casos. No obstante, también se pueden realizar extensiones al modelo de referencia pero con la pérdida correspondiente de los beneficios que reporta cualquiera de estas dos versiones.

Finalmente, siete actividades de control conforman el modelo de actividad de control: gestión de recetas, planificación y organización de la producción, gestión de la información de producción, gestión de célula, supervisión de unidad, control de proceso y, protección ambiental y del personal (fuera del ámbito de este estándar). Dichas actividades definen cómo se controlan los procesos por lotes empleando equipos $\mathrm{y} / \mathrm{o}$ personal.

Por tanto, al igual que GEMMA, S88 permite sistematizar la búsqueda de todas las situaciones que provocan la puesta en marcha, el paro, la entrada en producción, la presencia de errores... de un proceso automatizado. No obstante, la semántica de S88 se centra en las máquinas automatizadas, mientras que la guía GEMMA utiliza una semántica muy próxima al personal de planta.

En [18] se presenta la aplicación del estándar ISA-88 en distintos procesos de fabricación discreta y continua demostrando los beneficios de su aplicación. En esta recopilación de trabajos se presentan diferentes interpretaciones para la aplicación de la misma. En [4] se concluye con esta misma idea, indicando como el estándar es genérico y está abierto permitiendo numerosas interpretaciones.
Algunos trabajos han utilizado ISA-88 junto con otros estándares en el campo de la automatización. En [14] se realiza un análisis de compatibilidad con IEC 61499, en [10] se combinan ambos estándares para la implementación del control de procesos Bach. Trabajos posteriores siguen mostrando el interés por el estándar y la necesidad de su evolución. Así por ejemplo en [6] se plantea que la aplicación del S88 produce una repetición de la información en las recetas y un nivel bajo de reutilización. Para solucionar el problema planean nuevos modelos.

\section{$2.3 \quad$ S88 vs GEMMA}

La relación entre los 17 estados propuestos por la guía GEMMA y los 19 estados propuestos por la norma ANSI/ISA S88 se presenta en la Tabla 1.

Tabla 1. Relación de Estados

\begin{tabular}{|c|l|}
\hline $\begin{array}{c}\text { ANSI/ISA } \\
\mathbf{8 8}(\mathbf{2 0 1 0})\end{array}$ & \multicolumn{1}{|c|}{ GEMMA de ADEPA } \\
\hline STOPPED & A1. Paro en estado inicial \\
\hline RESETTING & A6. Inicialización de la parte operativa \\
\hline IDLE & \\
\hline STARTING & F2. Marcha de preparación \\
\hline RUNNING & $\begin{array}{l}\text { F1. Producción normal } \\
\text { F5. Verificación de marcha en orden }\end{array}$ \\
\hline COMPLETING & F3. Marcha de finalización \\
\hline COMPLETE & \\
\hline STOPPING & A4. Paro en estado intermedio \\
\hline ABORTING & D1. Paro de emergencia \\
\hline ABORTED & \\
\hline CLEARING & $\begin{array}{l}\text { D2. Diagnóstico y/o tratamiento del fallo } \\
\text { A7.Preposicionamiento de la P.O. } \\
\text { A5. Preparación de arranque tras un fallo }\end{array}$ \\
\hline SUSPENDING & D2. Diagnóstico y/o tratamiento del fallo \\
\hline SUSPENDED & \\
\hline UNSUSPENDING & A5. Preparación de arranque tras fallo \\
\hline HOLDING & D2. Diagnóstico y/o tratamiento del fallo \\
\hline HELD & \\
\hline UNHOLDING & A5. Preparación de arranque tras fallo \\
\hline PAUSING & $\begin{array}{l}\text { A3. Demanda de paro en estado } \\
\text { intermedio }\end{array}$ \\
\hline PAUSED & A4. Paro en estado intermedio \\
\hline & A2. Demanda de paro al final de ciclo \\
\hline & F4. Verificación de marcha en desorden \\
\hline D3. Seguir en producción con fallo \\
\hline & F6. Marcha de test \\
\hline
\end{tabular}

Como ya se ha comentado, el diagrama de transición de estados de S88 incluye dos tipos de estados: los estado de acción y los estados de espera (resaltados en la Tabla 1) en los que se mantiene su condición hasta que se produzca la transición a otro estado de acción. Sin embargo, la guía GEMMA únicamente propone dos estados de espera: A1 - Paro en estado inicial, que corresponde al estado inicial de reposo de la parte operativa, y A4 - Paro en estado intermedio. Para los restantes estados de espera de S88 no existe correspondencia con estados de GEMMA. En cuanto a los estados de acción, las relaciones son "uno a uno", "uno a varios" y "varios a uno". 
Así, por ejemplo, el estado CLEARING presenta una relación "uno a varios". Este estado agrupa las acciones a realizar tras el paro de emergencia, como son: el diagnóstico de la emergencia para averiguar el origen y el tratamiento de la misma (por ejemplo, paso al modo manual para realizar un tratamiento específico), las acciones específicas para asegurar el estado de ciertos elementos del proceso (por ejemplo, posición de los elevadores, pinzas o mordazas abiertas o cerradas, cilindros extendidos o recogidos...) y las acciones a la salida de la emergencia (por ejemplo, operaciones de vaciado y/o limpieza de alguna máquina, calibración...). Por tanto, dicho estado englobaría tres estados GEMMA: D2 - Diagnóstico y/o tratamiento del fallo, A7 Preposicionamiento de la Parte Operativa y 15 Preparación de arranque tras fallo.

Los estados SUSPENDING y HOLDING se activan al producirse un fallo diferenciándose en la realización de las acciones con o sin intervención del personal de planta. Dichos estados constituyen un ejemplo de una relación "varios a uno", ya que en ambos casos la correspondencia es con el estado D2 Diagnóstico y/o tratamiento del fallo de GEMMA.

Los estados A2, F4, D3 y F6 de GEMMA no presentan relación con ningún estado S88. Algunos estados no se consideran necesarios al ser un estándar derivado del proceso por lotes; por ejemplo: el estado A2 - Demanda de paro a fin de ciclo, ya que en estos procesos el final del ciclo lo marca el lote, o el estado F4 - Verificación de marcha en desorden en el que la máquina puede realizar cualquier movimiento (o unos movimientos preestablecidos) normalmente por orden del operador, para realizar tareas de mantenimiento y verificación, permitiendo verificar individualmente ciertos movimientos o partes del proceso, normalmente en vacío (sin producir), sin respetar el orden habitual del ciclo.

No obstante, el estándar presenta los estados principales y queda abierto a la definición de estados adicionales para su adaptación al control de los procesos de fabricación discreta, procesos continuos e incluso en logística.

\section{HACIA MeiA. 4.0}

La metodología MeiA combina la madurez de las disciplinas de la ingeniería de software con los métodos y estándares del campo de la automatización industrial. En cada una de sus seis fases se analiza una parte del sistema de control de manera "independiente": la Fase I establece la secuencia principal del funcionamiento del sistema en modo automático; las Fases II y III organizan tanto el arranque como la parada del sistema en modo manual y pruebas, respectivamente; la Fase IV identifica, analiza y evalúa los fallos que pueden producirse en el proceso; la Fase V organiza tanto el arranque como la parada del sistema para el tratamiento de emergencias; y finalmente, la Fase VI establece el ciclo de producción.

Cada fase consta de un conjunto de pasos que son los que guían el proceso de análisis y diseño para obtener GEMMA y los Casos de Uso, así como los DOUs (Unidades de Organización de Diseño; Design Organization Units) y las señales de coordinación entre ellos. Además, ayuda a identificar las necesidades de supervisión/control, y a configurar el Panel de Operación así como otros paneles auxiliares con accionamientos e indicadores específicos.

En los procesos de diseño, MeiA incorpora el lenguaje de modelado GRAFCET para generar las unidades de organización de diseño DOUs. Se distinguen tres tipos: DOUs de decisión que organizan el arranque y la parada de los distintos modos de funcionamiento, y coordinan todos los posibles estados del sistema; DOUs de producción que realizan las operaciones de producción, coordinación de operaciones, selección de parámetros de producción, etc; DOUs auxiliares que realizan procedimientos de inicialización, preparación, paros, avisos, etc.

La extensión de la metodología MeiA dos aspectos. Por un lado, se ha establecido la relación entre los modelos obtenidos siguiendo la metodología MeiA y los modelos propuestos por S88 (ver Figura 1).

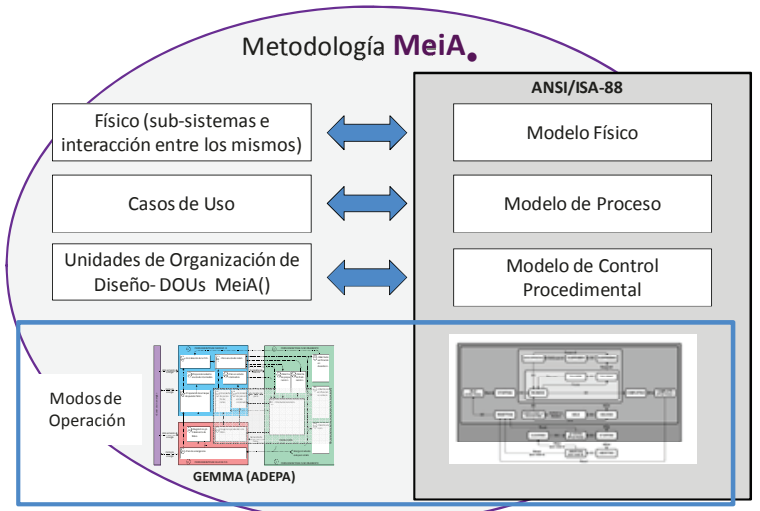

Figura 1. Relación de MeiA• con los Modelos de S88

Por otro lado, para la consideración de los distintos modos de operación de acuerdo al estándar S88, se ha realizado un análisis de todos los estados. El resultado de este análisis se muestra en la Tabla 2 donde se presenta la relación de los estados de S88 con la propuesta de estados de GEMMA que se realiza en la metodología MeiA. Al establecer esta relación surge la necesidad de incluir nuevos estados (EndCycle, TestRunning, FailRunning, 
ManualRunning, ReStarting, ReCompleting y Diagnosing) que recogen las necesidades de los sistemas de control de los procesos de fabricación discreta y procesos continuos. Partiendo de la tabla se han realizado las plantillas con los Modelos de Estados de cada Fase. Así, por ejemplo, en la Figura 2 se presenta el Modelo de Estados para la Fase I.

Los seis pasos de análisis de la Fase I permiten identificar los estados en $\mathbf{S 8 8}$ que contempla la secuencia principal, y los comandos que permiten la evolución entre estos estados. Así, partiendo del sistema en el estado Stopped, al solicitar su funcionamiento automático, se pasará al estado Reseting donde se deberán verificar las condiciones iniciales y de seguridad del sistema. De este estado se pasará al estado Ilde, y aquellos sistemas que requieran tareas de preparación previas a la producción, pasarán al estado Starting como paso previo al estado Running. Cuando el sistema se encuentra en producción y se solicita la parada a fin de ciclo, el sistema pasará al estado EndCycle hasta que se finalice el ciclo. Aquellos sistemas que precisen operaciones adicionales una vez finalizada la producción, evolucionarán al estado Completing, tras lo cual el sistema pasará al estado Completed y se detendrá en el estado de reposo Stopped.

Si en cualquier momento se solicita el Paro del Proceso (comando Stop), el sistema pasará al estado Stopping y tras la detención evolucionará al estado Stopped.
Tabla 2. Relación de Estados

\begin{tabular}{|c|l|}
\hline MeiA. S88 & \multicolumn{1}{|c|}{ GEMMA } \\
\hline STOPPED & A1. Paro en estado inicial \\
\hline RESETTING & A6. Inicialización de la parte operativa \\
\hline IDLE & \\
\hline STARTING & F2. Marcha de preparación \\
\hline RUNNING & F1. Producción normal \\
\hline ENDCYCLE & A2. Demanda de paro al final de ciclo \\
\hline COMPLETING & F3. Marcha de finalización \\
\hline COMPLETE & \\
\hline STOPPING & A4. Paro en estado intermedio \\
\hline ABORTING & D1. Paro de emergencia \\
\hline ABORTED & \\
\hline CLEARING & D2. Diagnóstico y/o tratamiento del fallo \\
\hline SUSPENDING & D2. Diagnóstico y/o tratamiento del fallo \\
\hline SUSPENDED & \\
\hline UNSUSPENDING & $\begin{array}{l}\text { A5. Preparación de arranque después de: } \\
\text { Fallo, Emergencia, Manual }\end{array}$ \\
\hline HOLDING & D2. Diagnóstico y/o tratamiento del fallo \\
\hline HELD & \\
\hline UNHOLDING & $\begin{array}{l}\text { A5. Preparación de arranque después de: } \\
\text { Fallo, Emergencia, Manual }\end{array}$ \\
\hline PASTRUNING & $\begin{array}{l}\text { A3. Demanda de paro en un estado } \\
\text { intermedio }\end{array}$ \\
\hline FAILRUNNING & A4. Paro en estado intermedio \\
\hline MANUALRUNNING & F4. Verificación de marcha en orden \\
\hline RESTARTING & $\begin{array}{l}\text { A7.Preposicionamiento de la parte } \\
\text { operativa }\end{array}$ \\
\hline A5. Preparación de arranque después de: \\
Fallo, Emergencia, Manual \\
\hline D2. Diagnóstico y/o tratamiento del fallo \\
\hline DAMLETING
\end{tabular}

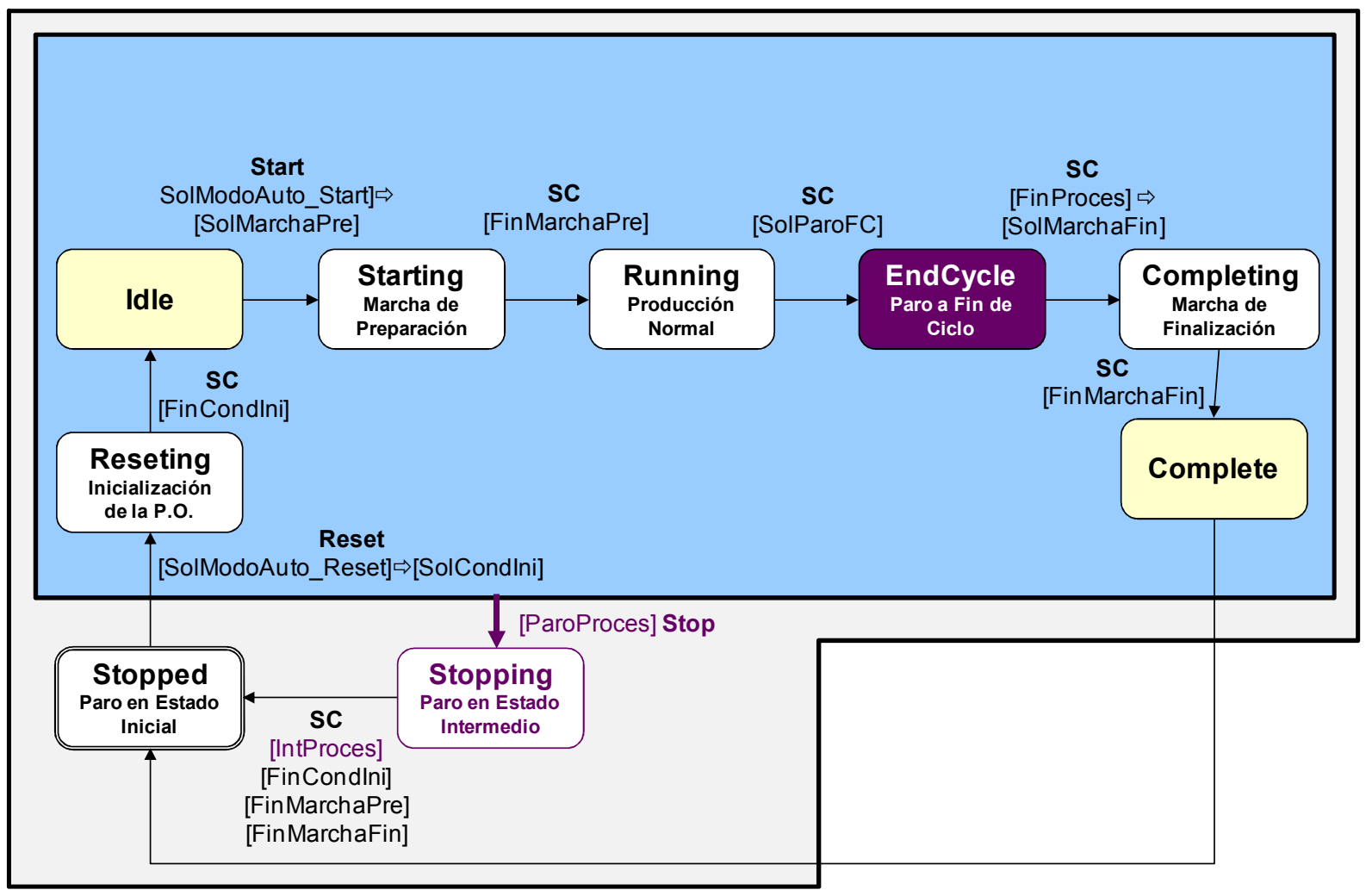

Figura 2. Plantilla Modelo de Estados S88 para las Fase I-Secuencia Principal 


\section{CASO DE ESTUDIO}

En este apartado se presenta un caso de estudio que aborda el desarrollo del software de control de la célula de ensamblaje DISA FMS200 ubicada en el departamento de Ingeniería de Sistemas y Automática de la Escuela de Ingeniería de Bilbao. La célula de ensamblaje DISA FMS200 consta de 5 estaciones y un sistema de transporte encargado de gestionar el movimiento de palets entre las distintas estaciones. En la célula se ensambla un conjunto formado por cuatro piezas (base, rodamiento, eje y tapa) pudiéndose montar diferentes conjuntos combinando diferentes tipos de piezas.

En la Figura 3 se presenta una fracción del Modelo Físico del sistema según el estándar S88. La Célula FMS200 contiene seis Unidades que se corresponden con cada una de las estaciones y el sistema de transporte. A su vez, cada una de ellas, puede contener un conjunto de Módulos de Equipo y Módulos de Control. Así, por ejemplo, la Estación 1Ubicación Base dispone de 6 Módulos de Equipo y un Módulo de Control.

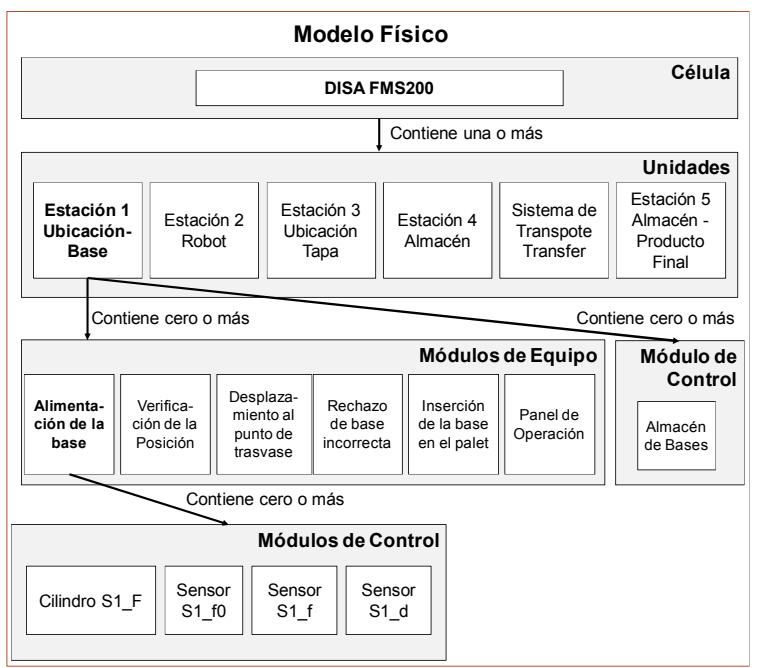

Figura 3. Modelo Físico DISA FMS200

En la Figura 4 se muestra una parte del Modelo del Proceso según el estándar S88. El proceso consiste en el ensamblado de conjuntos, y las operaciones para su realización se definen en las Etapas del Proceso que operan de forma independiente entre ellas. Dentro de estas Etapas se distinguen dos grupos: las que definen las operaciones de producción y las que especifican las funciones de mando (enmarcadas en color azul). Las Etapas del Proceso a su vez se dividen en Operaciones que representan las actividades especializadas de procesamiento. Por último, las Operaciones se subdividen en un conjunto de Acciones para llevar a cabo el procesamiento requerido por cada operación de proceso.

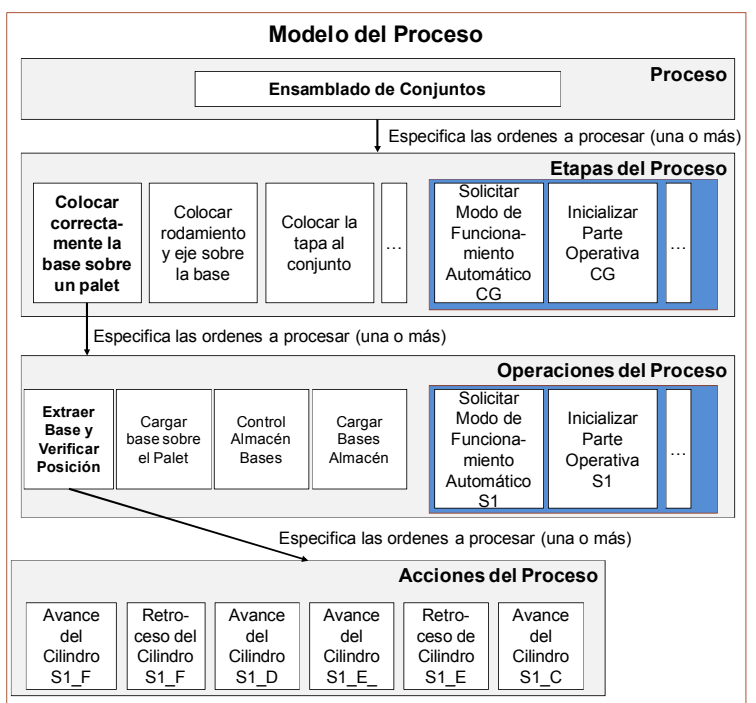

Figura 4. Modelo de Proceso Ensamblado de Conjuntos

Para el Modelo de Control Procedimental según el estándar S88, en el nivel de Procedimiento se encuentra el Control General de la Célula que por medio de los Procedimientos de Unidad ejecuta las operaciones de producción atendiendo a las distintas formas de mando para los sistemas de control de las estaciones y del sistema de transporte. A su vez cada Procedimiento de Unidad realiza un conjunto de Operaciones de producción que son desarrolladas en más detalle por las Fases.

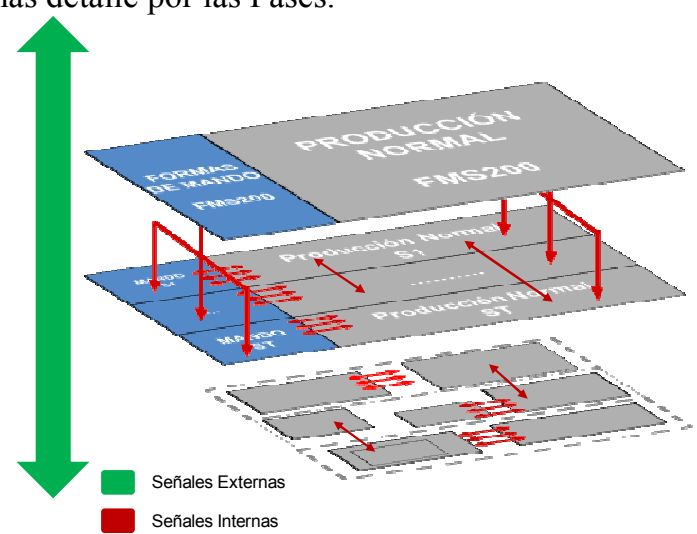

Figura 5. Niveles del Sistema de Control

En la Figura 5 se ilustran los niveles del Sistema de Control de manera esquemática. En cada nivel se distinguen dos zonas que representan la gestión de las formas de mando y la gestión de las operaciones de producción. En el nivel superior se sitúa el Control General, en el nivel intermedio los sistemas de Control de las Estaciones y del sistema de transporte, y en el nivel inferior una definición más precisa de las operaciones de producción.

En lo que respecta a los modos de operación, la Figura 6 muestra el Diagrama de Estados para la Fase I de la Estación 1-Ubicación Base relacionando los estados de GEMMA con los estados de S88. 


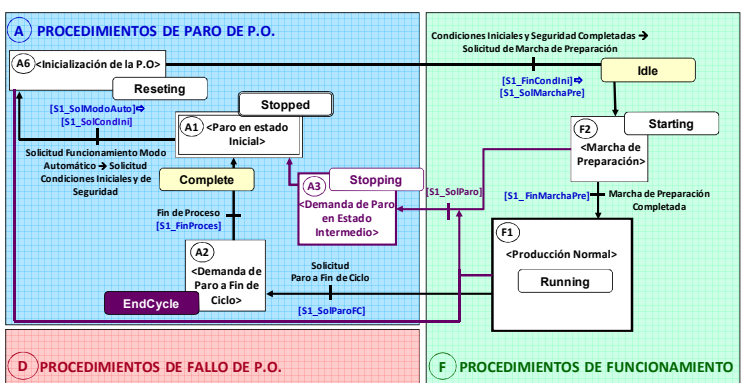

Figura 6. Diagrama de Estados para la Fase I de la Estación 1-Ubicación Base

\section{CONCLUSIONES}

La metodología MeiA integra la madurez de las disciplinas de la ingeniería del software con los métodos y estándares extendidos en el ámbito de la automatización para desarrollar sistemas de control de una manera estructurada y bien documentada de calidad con menores tiempos de implementación.

No obstante, en este trabajo se ha extendido para incorporar el estándar ANSI/ISA-88 propuesto en el marco de Industry 4.0 en lo que respecta al proceso productivo, de modo que se obtienen los modelos propuestos por el estándar siguiendo la metodología, así como la descripción de los modos de operación de acuerdo a dicho estándar.

El trabajo futuro contempla utilizar los principios de MDE para generarlos automáticamente.

\section{Agradecimientos}

Este trabajo está financiado por el proyecto DPI201568602-R del Gobierno Español y el proyecto UFI11/28 de la UPV/EHU.

\section{Referencias}

[1] ADEPA - Agence nationale pour le DEveloppment de la Production Automatisée (1981) "GEMMA (Guide d'Étude des Modes de Marches et d'Arrêts)".

[2] AFCET (Association Française pour la Cybernétique Economique et Technique) Commission, (1977) "Normalisation de la Représentation du Cahier des Charges d'un Automatisme Logique“, Technical report.

[3] DIN/DKE - Deutsches Institut für Normung / Deutsche Kommission Elektrotechnik (2016) "German Standardization Roadmap. Industry 4.0", disponible en www.din.de/go/roadmapindustrie40-en.

[4] D. James, (2006) "Batch of the day [batch processing]", Computing and Control Engineering, vol. 17, no 4, pp. 30-35.

[5] González, V.M., Mateos F., Ng A., (2004) "MLAV: the Object-oriented Methodology of the Virtual Automation Lab", IEEE International Conference on Robotics and Automation, New Orleans (USA).

[6] G. Godena, T. Lukman, I. Steiner, F. Bergant y S. Strmcnik, (2015) "A new object model of batch equipment and procedural control for better recipe reuse",v Computers in Industry, vol. 70, p. 46-55.

[7] IEC 60848, (2002) "GRAFCET specification language for sequential function charts", International Electrotechnical Commission.

[8] ISA, (2010) “ANSI/ISA-88.00.01-2010. Batch Control".

[9] ISO/IEC 12207, (2008) "Systems and software engineering - Software life cycle processes". International Organization for Standardisation,

[10] Ivanova, D, y otros, (2009) "Combining IEC 61499 and ISA S88 for Batch Control", Moscow, Russia.

[11] Jacobson, I., (1992) "Object Oriented Software Engineering. A Use Case Driven Approach", Addison Wesley.

[12] Machado, J. and Seabra, E., (2010) "A systematized approach to obtain dependable controllers specifications", ABCM Symposium Series in Mechatronics, vol. 4, pp. 408-417.

[13] Ministerio de Industria, Energía y Turismo, (2014) "Industria Conectada 4.0 - La transformación digital de la Industria Española”, disponible en www.industriaconectada40.gob.es.

[14] M. Sousa, (2010), "Analyzing the compatibility between ISA 88 and IEC 61499", Emerging Technologies and Factory Automation (ETFA), pp. 1 - 8.

[15] M. L. Álvarez, E. Estévez, I. Sarachaga, A. Burgos y M. Marcos (2013) "A novel approach for supporting the development cycle of automation systems", International Journal of Advanced Manufacturing Technology, vol. 68, pp. 711-725.

[16] Ponsa, P., Vilanova, R., Díaz, M., (2006) "Introduction of the human operator into the automation cycle with the GEMMA guide", International Control Conference, Glasgow (Scotland), pp. 1-6.

[17] S. Panjaitan, (2010) "A Formal Design of Automation Systems based on Operation Modes using High-level Petri Net", Second International Conference on Advances in Computing, Control, and Telecommunication Technologies, pp. 129-131.

[18] WBF Conference, (2010), “Applying ISA-88 in Discrete and Continuous Manufacturing”, vol. 2, N. York, Ed., Momentum Press. 